\title{
Effect of Nanofiller Shape on Effective Thermal Conductivity of Fluoropolymer Composites
}

\author{
Dylan Smith ${ }^{1}$, Michelle Pantoya $^{1 *}$ \\ ${ }^{1}$ Department of Mechanical Engineering, Texas Tech University, Lubbock, TX 79409 \\ *Corresponding author contact information: michelle.pantoya@ttu.edu phone: 806-834-3733
} Abstract

Filler particle shape and size influence interconnectivity within a polymer matrix and both play a significant role in controlling the effective thermal conductivity of a composite. This study examines the effect of nanofiller particle shape in a polytetrafluorethylene (PTFE) matrix on thermal energy transport. Experimental measurements using a laser flash analysis method are performed on PTFE with three different shaped carbon fillers: nano-diamond spheres, carbon nanotubes (CNT) and graphene flakes. Filler concentration ranged from 0 to $30 \%$. The experimental results are coupled with a particle connectivity model to understand the relationship between energy transport and filler connectivity. Results show filler particles with more micron scale dimensions form better interconnected networks at lower volume concentrations than fillers with fewer micron scale dimensions. Filler connectivity influences effective conductivity: specifically, nano-diamond spheres showed negligible interconnectivity and result in negligible change in thermal conductivity. The CNT, with one-micron scale dimension (i.e., length) and flakes, with two-micron scale dimensions (i.e., length and height), both exhibited linear increases in interconnectivity and effective thermal conductivity. Graphene interconnectivity in PTFE is six times that of CNT and thermal conductivity is $0.5 \mathrm{~W} / \mathrm{mK}$, two times that of CNT, respectively. These results provide new insights on how to optimize nanoparticle structure to enhance thermal energy transport.

KEY WORDS: Carbon nanotubes; Nano particles; Thermal properties; Polymer-matrix composites (PMCs); Modelling 


\section{Introduction}

Fluoropolymers are generally poor conductors of thermal energy, yet offer many other desirable properties such as low cost, ease of manufacturing and good mechanical properties such as wear resistance[1]. The effective thermal properties of a fluoropolymer can be controlled by adding a small concentration of conductive material, called filler. In this way, the fluoropolymer matrix and filler produce a composite with the advantages of a fluoropolymer plus tailored thermal properties associated with the filler. One approach is to identify a highly conductive filler and calculate an optimal effective thermal conductivity based on a weighted average of the matrix plus filler. But, the weighted average approach often does not correspond well with measured effective thermal conductivity[2]. For example, Biercuk et al. added singlewall carbon nanotubes (SWNT) and vapor grown carbon fibers to augment the thermal transport properties of industrial epoxy[3]. They predicted that carbon fibers would increase effective thermal conductivity $100 \%$ more than the SWNT based on a weighted average estimate. In contrast, experimental results showed thermal conductivity only increased $45 \%$ in carbon fiber composites but increased an astounding $125 \%$ with $1.0 \mathrm{wt} . \%$ SWNT. They suggested that the lager aspect ratio of the SWNT compared to the carbon fibers formed a percolation network at loading[3]. In another study, Weidenfeller et al. [4] used copper and talc fillers in a polypropylene (PP) matrix to increase effective thermal conductivity. The bulk thermal conductivity of copper is forty times greater than talc and a weighted average model predicted that the thermal conductivity of $\mathrm{PP} / \mathrm{Cu}$ would be significantly greater than $\mathrm{PP} /$ talc. But, at 30 vol. \% filler, results showed $\mathrm{PP} / \mathrm{Cu}$ effective thermal conductivity was half that of $\mathrm{PP} /$ talc. Weidenfeller developed an "interconnectivity model" which was used to explain the lower 
conductivity in the $\mathrm{PP} / \mathrm{Cu}[4]$. These studies indicate that thermal properties alone do not control the effective conductivity of a heterogeneous composite.

Another form of the weighted average approach was proposed by Nielsen et al. [5] for a mixture with two continuous phases and is given in Eq. (1).

$$
P^{n}=P_{A}^{n} X_{A}+P_{B}^{n} X_{B}
$$

In this model, $P$ is a property of the material such as elastic modulus, dielectric constant, or thermal conductivity and $X$ is volume concentration of phase $A$ or $B$, respectively. In Eq. (1), the mixing parameter $n$ is a function of the morphology (size, shape, and orientation) and the type of property being measured. If morphology is the same for both phases, $n$ is constant and has been shown to vary based on the property being measured. Davies et al. showed $n=1 / 3$ for the dielectric constant and $n=1 / 5$ for elastic modulus [5]. When $n=1$, Eq. (1) results in the ordinary rule of mixtures; when $n=-1$, the inverse rule of mixtures; and, when $n=0$, a logarithmic rule of mixtures is obtained. If morphology is considered, the value of $n$ varies with volume concentration. To the authors' knowledge this model has not been applied to thermal conductivity, yet Eq. (1) is proposed to apply to thermal conductivity [5].

The idea that filler size, shape, orientation and concentration have a significant influence on effective thermal conductivity of a composite is not new [6-13]. Wang et al.[9] and Zhang et al. [8] developed heat transfer simulations to examine filler size and shape influences on effective thermal conductivity. Zhang et al. found that an I shape had the greatest thermal conductivity [14] while Wang et al.[9] found that a Double Y shape had an even greater increase in thermal conductivity. They both showed that thermal conductivity is affected by the orientation angle of the fillers. For example, if the longest filler dimension is parallel to the 
direction of heat flow, the filler enhanced effective thermal conductivity up to $100 \%$ more than if the longest filler length was perpendicular to the direction of heat flow[8].Also, Liang et al. found that effective thermal conductivity increased with filler size in $\mathrm{PP} / \mathrm{Al}(\mathrm{OH})_{3}$ and $\mathrm{PP} / \mathrm{Mg}(\mathrm{OH})_{2}$ composites [10]. They looked at sizes ranging from 1 to $10 \mu \mathrm{m}$ and showed an increase in effective thermal conductivity of $13 \%$ [9]. Zhang et al. [7] found that thermal conductivity increased by $50 \%$ with filler size in $\mathrm{Al}_{2} \mathrm{O}_{3}$ ranging from $0.1 \mu \mathrm{m}$ to $10 \mu \mathrm{m}$ in diameter. With all of these studies there still exists a gap in quantifying the effect of size and shape as a function of concentration and relate that to effective conductivity in a way that accurately predicts effective conductivity.

The objective of this study is to examine the effects of dimensionality for nanoparticle fillers on connectivity through a matrix and the resulting effective thermal conductivity of a composite. This objective will be accomplished using a model that describes filler connectivity coupled with experimental thermal property measurements of polytetrafluoroethylene (PTFE) as a base matrix combined with three different morphologies of a carbon powder filler. The filler particles include rectangular graphene flakes, carbon nanotubes (CNT), and spherical nanodiamond particles. Thermal properties measurements were performed for compacted powders using a laser flash analyzer (LFA). Also, the applicability of Eq. (1) to the matrix-filler composites studied here was examined and new $n$ values applicable for thermal conductivity were determined as a function of filler morphology.

\subsection{Interconnectivity}

Quantifying filler interconnectivity was done using the Hashin-Shtrikman (HS) model $[15,16]$. This method defines an upper and lower bound based on a transport coefficient (i.e. 
thermal conductivity) and the volume percent filler and assumes a two-phase composite with one phase a polymer matrix $(M)$ and the second phase spherical filler particles $(F)$ with no porosity within the composite. The expression for the upper bound is given by Wagner[17] for electrical conductivity and later modified for thermal conductivity [18]. The lower bound describes a composite with effective thermal conductivity limited by the lower value of thermal conductivity (i.e., PTFE) with fillers of higher thermal conductivity (i.e., carbon). The upper bound describes a composite with effective thermal conductivity limited by a matrix of higher thermal conductivity (carbon) with filler of lower thermal conductivity (i.e., PTFE). The expressions for the upper and lower bound are given in Eq. (2) and (3) [18].

$$
\begin{aligned}
& \text { Upper Bound }=\lambda^{H S+}=\lambda_{F} \frac{2 \lambda_{F}+\lambda_{M}-2 X_{M}\left(\lambda_{F}-\lambda_{M}\right)}{2 \lambda_{F}+\lambda_{M}+X_{M}\left(\lambda_{F}-\lambda_{M}\right)} \\
& \text { Lower Bound }=\lambda^{H S-}=\lambda_{M} \frac{2 \lambda_{M}+\lambda_{F}-2 X_{F}\left(\lambda_{M}-\lambda_{F}\right)}{2 \lambda_{M}+\lambda_{F}+X_{F}\left(\lambda_{M}-\lambda_{F}\right)}
\end{aligned}
$$

In Eq.(2) and (3), $\lambda_{F}$ is the thermal conductivity of the filler, $\lambda_{M}$ is the thermal conductivity of the polymer matrix, and $X_{M}$ and $X_{F}$ are the volume concentration of the matrix and filler, respectively. Using the HS model for the upper and lower bounds, a value of interconnectivity is given by a method described by Schilling and Partzsch [19].

$$
X_{\text {interconnected }}=\frac{\lambda_{\text {Measured }}-\lambda^{H S-}}{\lambda^{H S+}-\lambda^{H S-}}
$$

In Eq. (4), $\lambda_{\text {Measured }}$ is the measured composite thermal conductivity. This method was modified to include the effects of air by estimating values of $\lambda_{\mathrm{F}}$ and $\lambda_{\mathrm{M}}$ from measurements taken from compressed powder pellets with similar bulk density.

\section{Experiment}

\subsection{Materials}


The composites consisted of DuPont Teflon® PTFE $60(\sim 5 \mu \mathrm{m}$ particle size $)$ matrix filled with varied concentrations of three different shapes of carbon fillers: graphene flakes, nanotubes (CNT), spherical diamond.

Graphene (Grade AO-2), Fig.1a, was supplied by Graphene Supermarket (Calverton, NY) and are irregularly shaped but approximated as rectangular with two lateral micron-scale dimensions ranging from 150-3000 $\mathrm{nm}$ and $8 \mathrm{~nm}$ thickness. The CNT, Fig.1b, was supplied by Alfa Aesar; Ward Hill, MA) with a 3-20 nm outside diameter with 1-3 nm inside diameter and a 0.1-10 $\mu \mathrm{m}$ length. The diamond nanoparticles, Fig. 1c, were supplied by Skyspring Nanomaterials (Houston, TX) and are spherical with a 3-4 nm diameter.
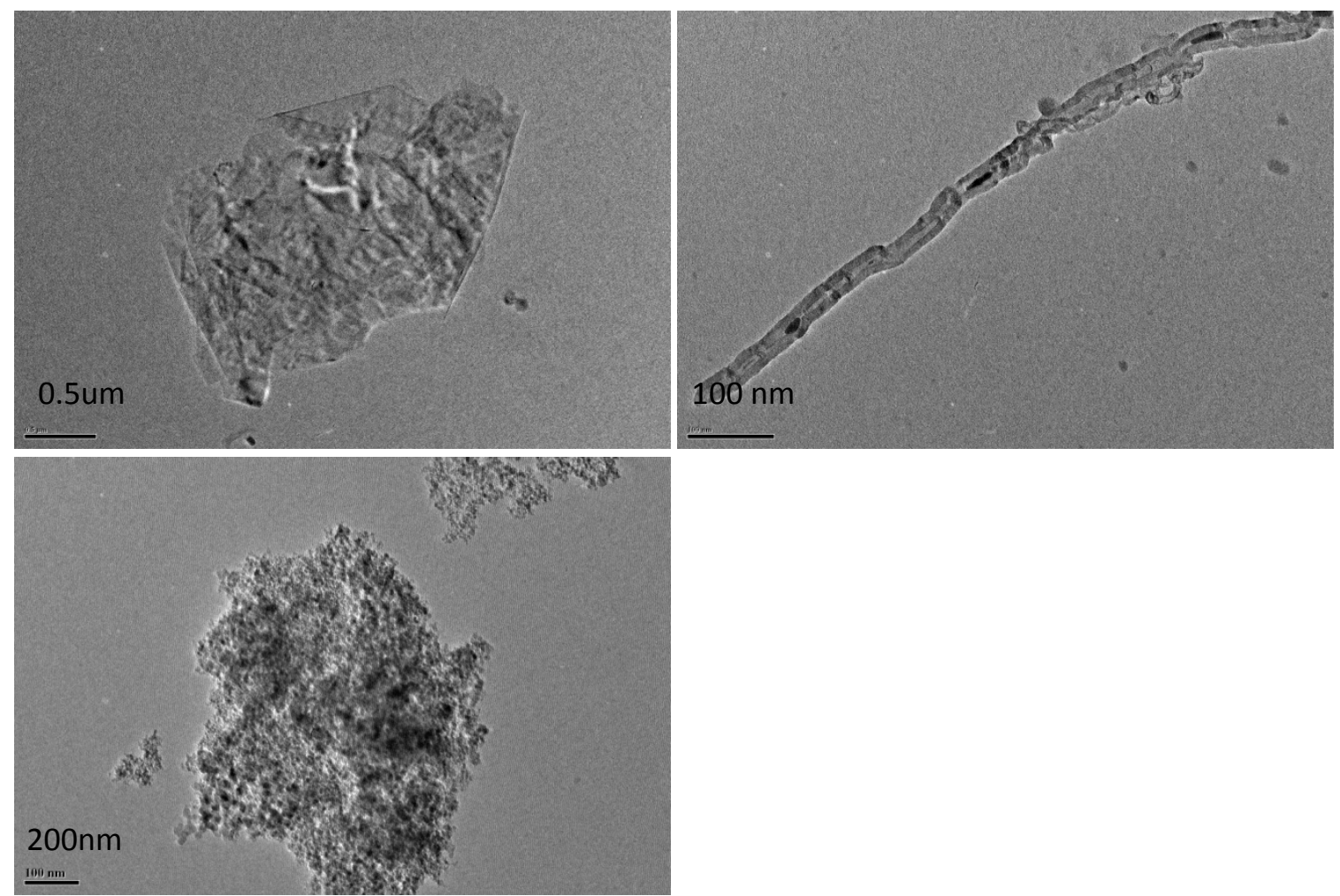

Figure 1. SEM images of a) graphene b) CNT c) diamond aggregate 
Five different filler concentrations ranging from 0 to 30 vol. $\%$ were calculated with supplier specified material densities and controlled volume to achieve a constant bulk density of $1.1 \mathrm{~g} / \mathrm{cm}^{3}$ with and accuracy of $5 \%$. A maximum concentration of $20 \mathrm{vol} . \%$ was made for diamond because beyond that concentration, the pellets became fragile and did not maintain constant dimensions. The filler and PTFE were weighed and mixed with a shaker until further mixing did not change mixture appearance. The powder was cold pressed into a cylinder pellet with $12.6 \mathrm{~mm}$ diameter and $1.7 \mathrm{~mm}$ thickness using a die designed to facilitate pressing powders with a hydraulic press. Pellets were weighed and measured. Using the thickness of $1.7 \mathrm{~mm}$ and diameter of $12.6 \mathrm{~mm}$, the volume of each pellet was calculated. The bulk density was then calculated using the measured mass and volume of each pellet. The pellets were then coated with a dry graphite film lubricant to facilitate absorption of incident radiation.

\subsection{Laser Flash Analyzer}

Thermal diffusivity measurements were made with a Netzsch Laser Flash Analyzer (LFA) 447 at $25^{\circ} \mathrm{C}$ for each sample. The Netzsch LFA 447 works on the flash diffusivity method. In this method, a xenon flash lamp placed inside a parabolic mirror emits a 115 or 230 $\mathrm{V}, 50 / 60 \mathrm{HX}$, and $10 \mathrm{~A}$ short light pulse onto the sample. The time it takes for the sample to heat up is measured by a liquid-nitrogen cooled indium antimonide infrared detector. From this thermal diffusivity is calculated by Eq. (5)[20].

$$
\alpha=13.88 \frac{l^{2}}{t_{50}}
$$

In this equation, $\alpha$ is the thermal diffusivity, $l$ is the thickness of the sample, and $t_{50}$ is the time corresponding to $50 \%$ of the temperature increase. The LFA uses a reference sample with a know $\mathrm{Cp}$ value and Eq. (6) to calculate the $\mathrm{Cp}$ of the sample 


$$
C_{P_{\text {sample }}}=\frac{\left(m C_{p} \Delta T\right)_{r e f}}{(m \Delta T)_{\text {sample }}}
$$

The Pyroceram sample provided with the LFA was used to calculate The Cp values of the samples. With the diffusivity, $\mathrm{Cp}$, and density for each sample, the thermal conductivity of each sample was claculated using Eq. (7)

$$
\lambda=\alpha C_{p} \rho
$$

Where $\lambda$ is the thermal conductivity, $\mathrm{Cp}$ is the specific heat, and $\rho$ is the density. Five measurements for each sample were averaged for the final data. Analysis and determination of thermal conductivity was done with Netzsch Proteus software.

\section{Results}

\subsection{Thermal Conductivity}

Effective thermal conductivity measured for each filler shape and concentration are presented in Fig. 2. The effective thermal conductivity of PTFE/flake and PTFE/tube increased linearly with filler volume concentration and reached a maximum of 0.65 and $0.2 \mathrm{~W} / \mathrm{mK}$, respectively. The effective thermal conductivity of PTFE/diamond remained around the original value $0.07 \mathrm{~W} / \mathrm{mK}$ until 20 vol. \% filler where it increased to $1.0 \mathrm{~W} / \mathrm{mk}$. At greater than 20 vol.\% diamond filler, pellets with consistent dimensions could not be produced. The accuracy of the LFA measurements was lowest in the 30 vol. \% flake filler with a $3 \%$ difference between samples. The average difference between measurements was less than $2 \%$. 


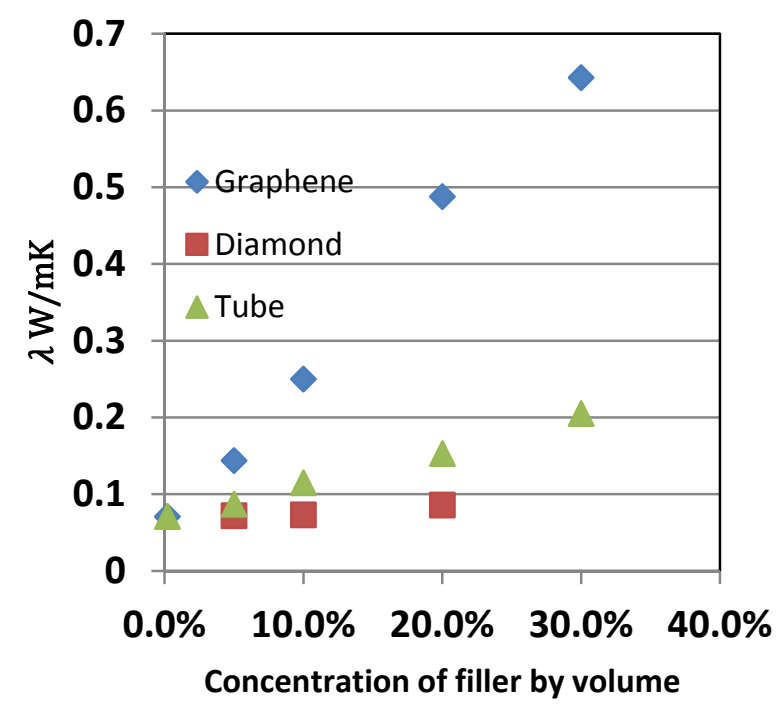

Figure 2. Measured effective thermal conductivity $(\lambda)$ of PTFE/Filler composites with different filler shapes and concentrations. Flakes indicated by diamond symbols, tubes indicated by triangle symbols and spheres indicated by square symbols.

\subsection{Interconnectivity}

To calculate filler interconnectivity, a pellet of pure PTFE at the same bulk density was prepared and the thermal conductivity was measured to be $0.06 \mathrm{~W} / \mathrm{mK}$. This was the value used for $\lambda_{M}$ in Eq.(2) and Eq.(3). The value for $\lambda_{F}$ was found similarly for graphene and was 2.0 $\mathrm{W} / \mathrm{mK}$. The results for interconnectivity are shown in Fig. 3 . 


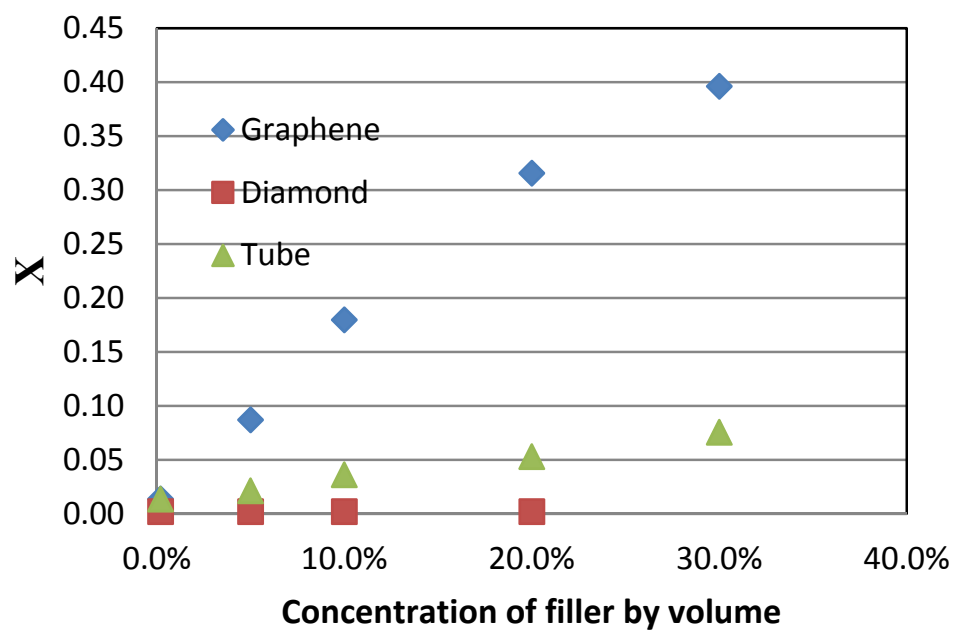

Figure 3. Calculated interconnectivity (X) using Eq. (2-4) for different shapes and concentration of filler.

Interconnectivity increased with filler concentration for flakes and tubes with a maximum of 0.4 and 0.075 at $30 \mathrm{vol} . \%$, respectively. Interconnectivity for diamond remained negligible up to 20 vol. \% filler. Interconnectivity for flakes was nearly six times greater than for tubes at all volume concentrations.

\subsection{Mixing Parameter}

The mixing parameter, $n$, in Eq. (1) provides a quantitative correction factor for a weighted average estimate of effective thermal conductivity. To calculate $n$ in Eq.(1), PTFE along with air void space is assumed to comprise a continuous matrix with a dispersed carbon filler. The measured thermal conductivity of the PTFE matrix (i.e., $0.06 \mathrm{~W} / \mathrm{mK}$ ) is used to determine the mixing parameter in Eq.(1). Thermal conductivity of the carbon filler is 1000 $\mathrm{W} / \mathrm{mK}$ and assumed constant for all shapes to isolate the influence of shape. Thermal conductivity of the three fillers is reported in the range of $25-5800 \mathrm{~W} / \mathrm{mK}[21-23]$. A constant 
value $1000 \mathrm{~W} / \mathrm{mK}$ is chosen because this value is in the range reported for all three shapes. The mixing parameter as a function of filler concentration is shown in Fig. 4.

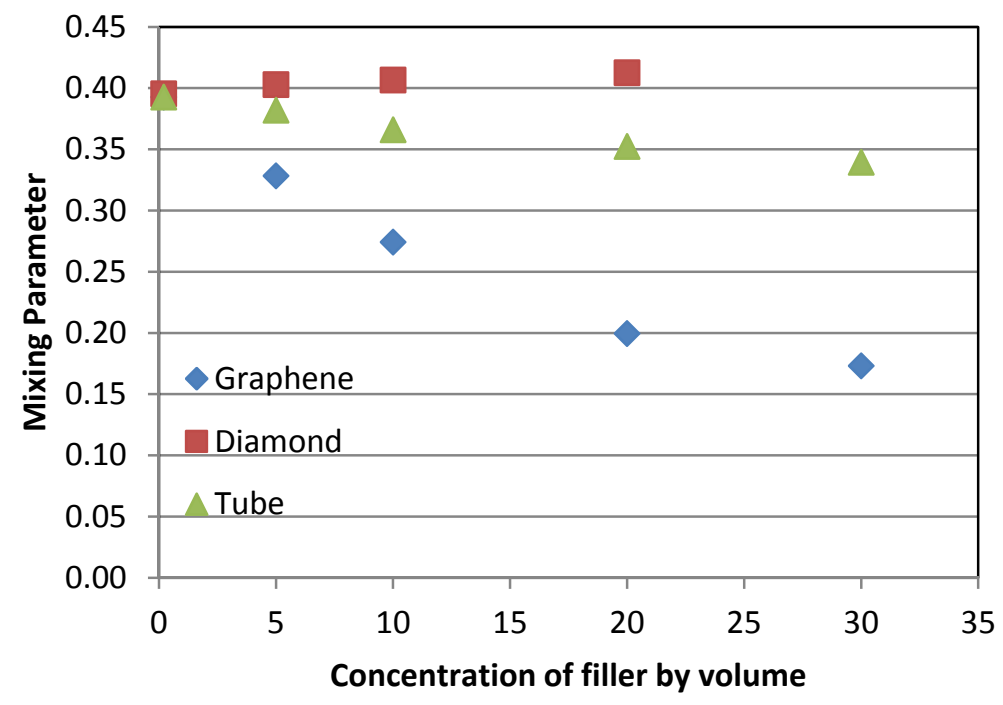

Figure 4. Mixing parameter ( $n$ ) as a function of filler concentration by volume percent and filler morphology.

The value of $n$ near 0 vol. $\%$ is 0.4 for all filler shapes and $n$ decreases as filler concentration increases for flakes and tubes, but remains constant for diamond. The value of $n$ decreases faster for graphene then for tubes with values of 0.17 and 0.34 at 30 vol. \%, respectively.

\section{Discussion}

The measured effective thermal conductivity shown in Fig. 2 are much lower than reported values of $0.25 \mathrm{~W} / \mathrm{mK}$ for PTFE and up to $5800 \mathrm{~W} / \mathrm{mK}$ for carbon [22]. This difference may be a result of the influence of air voids in the pellets which has a thermal conductivity significantly lower than PTFE or carbon (i.e., $0.025 \mathrm{~W} / \mathrm{mK}$ )[24]. The concentration of air voids 
in the samples can be assessed through the bulk density expressed in terms of percent theoretical maximum density (TMD). All pellets here have the same bulk density which corresponds to 50\% TMD such that half of each sample is air void space.

Interconnectivity is a measure of how well the filler particles connect throughout the PTFE/air matrix. At low filler concentrations, very few or no connected filler pathways are made through the matrix and the filler is considered a dispersed phase. At high filler concentration, multiple pathways are formed and combine to form a single, continuous pathway or phase. All fillers studied here are identified as nanoparticles because each has at least one dimension 100 $\mathrm{nm}$ or less. Figure 3 shows interconnectivity negligibly increased for diamond because the nanosized diameter did not allow for a continuous path of spherical particles to connect. The micronscale length of the CNT allowed some connectivity through the matrix. Graphene flakes have two micron-scale dimensions (i.e., length and height) while CNT only one (i.e., length) and the diamonds have none. The multiple micron-scale dimensions associated with the graphene flake morphology may be a key parameter influencing interconnectivity of the filler and explains the significant increase in thermal conductivity associated with graphene.

There is an inverse relationship between interconnectivity and the mixing parameter $n$ shown in Fig. 4. Nielson explains that not all materials attached to a continuous matrix act as a second continuous phase but can instead act as a dispersed phase [5]. The inverse relationship between interconnectivity and $n$ shows this. At 0.2 vol. \%, the filler for all three shapes acts like a dispersed phase in a continuous phase (i.e., PTFE). The two micron scale dimensions in graphene allow it to act more like a continuous a phase at lower concentration. The value of $n$ remains constant for diamond because with no micron-scale dimensions, the filler acts as a dispersed phase up to $20 \mathrm{vol} . \%$. Nielsen [4] noted that if the filler phase is continuous and 
randomly oriented, then a value of $n$ near zero would be expected. This is supported by the values of $n$ for graphene that decreases from 0.4 at 0.2 vol. \% filler to 0.17 at 30 vol. $\%$ filler, showing a decreasing value of $n$ as the filler phase becomes more continuous.

The shape of the thermal conductivity curve in Fig. 2 is interesting. Zhang et al. [6] showed that thermal conductivity as a function of concentration exhibits behavior that is analogous to percolation in electrical conductivity. It takes fewer connected pathways to effectively transfer electrical energy than thermal energy[25]. Because of this, there is no definite percolation concentration for thermal conductivity but instead a variation in thermal transport is indicated by an inflection point. At a certain filler concentration, there is an initial inflection point where there is a sharp increase in thermal conductivity and a second inflection point where the increase in thermal conductivity stabilizes [7]. Zhang et al. [7]showed that both inflection points occur at different concentrations for different filler sizes. In Zhang et al.[7], thermal conductivity was separated into three stages. The first stage describes a condition for no filler connected pathways (i.e. dispersed phase) and the increase in thermal conductivity is from the difference in thermal conductivity of the filler and PTFE as described by Eq.(1) with a constant $n$. The second stage is marked by the morphology of the filler particles that have a significant influence on the effective thermal conductivity by increasing the number of connected pathways which makes the filler act less like a dispersed phase and more like a continuous phase. In this stage, the value of $n$ in Eq.(1) changes as a function of filler concentration and is directly related to the change in interconnectivity(shown in Fig. 5). In the third stage, the filler has made enough connected pathways that it is no longer a dispersed phase but transitioned to a continuous phase. In this stage, PTFE and filler are both considered continuous phases, $n$ remains constant and the increase in effective thermal conductivity with concentration is from the material thermal 
properties, not the morphology. These inflection points are also related to interconnectivity.

Figure 5 shows a concept sketch of effective thermal conductivity with HS bounds.

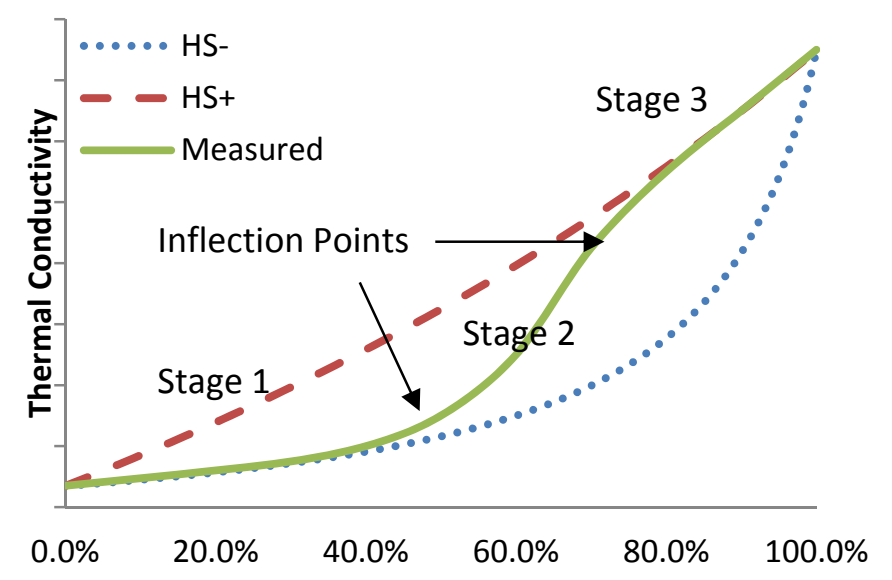

Figure 5. Concept sketch of effective thermal conductivity (solid line) and HS upper (longdashes) and lower (short dashes) bounds.

In Fig. (5), interconnectivity is smaller at the origin where the measured value is closer to the

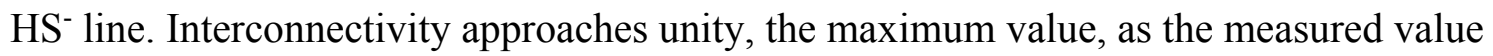
approached the $\mathrm{HS}^{+}$line. Interconnectivity increases the most in Stage 2 and is relatively stable in both Stage 1 and 3, which follows the same pattern as $n$ in Eq. (1). Measured values of interconnectivity could be used to predict the behavior of $n$ which will allow us to more accurately calculate the effective thermal conductivities of polymer composites from Eq. (1).

From Fig. 2, the inflection point for graphene was at very low concentration (i.e., 0.2 vol. $\%)$ and most of the measured values were in Stage 2. This low concentration for the inflection point makes sense because the two micron-scale filler dimensions create more connected pathways at lower concentrations. The first inflection point for CNT was between 5-10 vol. \%. The slope of the line in Stage 2 for CNT is smaller than for graphene. It is predicted that 
graphene, because of its earlier infection point and steeper slope, will reach its second inflection point at lower concentration than CNT because the two micron-scale lengths will form a continuous phase at lower concentrations. The smaller slope in Stage 2 of the CNT is because with only one micron-scale dimension, a higher filler concentration is needed to create interconnected pathways. The diamond filler never reached the inflection point which is shown by a constant $n$ value and interconnectivity of 0 .

\section{Conclusions}

The effects of filler shape on the interconnectivity and effective thermal conductivity of PTFE/carbon composites was studied. Below 20 vol. \% filler, 3-4 nm diameter spherical diamond particles show no interconnectivity. Graphene flakes with two micron-scale dimensions and CNT with one micron-scale dimension showed approximately linear trends below 30 vol. \% filler concentration. Graphene exhibited interconnectivity six times greater in the CNT, and an increase in effective thermal conductivity 3 times greater than CNT for 20 vol. \% filler concentration. Fillers with more micron scale dimensions form a more interconnected network through the composite. A modified weighted average model based on a mixing parameter was also presented. The mixing parameter was determined to be a function of both filler concentration and shape. These results show the importance of a continuous and dispersed phases on effective thermal conductivity and enable tailored thermal properties via selection of filler shape to control energy transport.

\section{Acknowledgements}

The authors are grateful for support from the Army Research Office grant number W911NF-14-1-0250 and encouragement from our program manager, Dr. Ralph Anthenien. 
Partial support is also provided by the Office of Naval Research under grant number N00014-12$1-0525$.

\section{References}

[1] J. Scheirs, Modern Fluoropolymers, John Wiley and Sons Inc., 1997.

[2] S. Duwe, C. Arlt, S. Aranda, U. Riedel, G. Ziegmann, A detailed thermal analysis of nanocomposites filled with $\mathrm{SiO} 2$, AlN or boehmite at varied contents and a review of selected rules of mixture, Compos. Sci. Technol. 72 (2012) 1324-1330. doi:10.1016/j.compscitech.2012.04.015.

[3] M.J. Biercuk, M.C. Llaguno, M. Radosavljevic, J.K. Hyun, a. T. Johnson, J.E. Fischer, Carbon nanotube composites for thermal management, Appl. Phys. Lett. 80 (2002) $2767-$ 2769. doi:10.1063/1.1469696.

[4] B. Weidenfeller, M. Höfer, F.R. Schilling, Thermal conductivity, thermal diffusivity, and specific heat capacity of particle filled polypropylene, Compos. Part A Appl. Sci. Manuf. 35 (2004) 423-429. doi:10.1016/j.compositesa.2003.11.005.

[5] L. Nielsen, Polymeric Composite Systems with two continuous phases, J. Appl. Polym. Sci. 21 (1977) 1579-1584.

[6] a Boudenne, L. Ibos, E. Gehin, M. Fois, J.C. Majeste, Anomalous behavior of thermal conductivity and diffusivity in polymeric materials filled with metallic particles, J. Mater. Sci. 40 (2005) 4163-4167. <Go to ISI>://000231417300001.

[7] S. Zhang, The effects of particle size and content on the thermal conductivity and mechanical properties of A12O3/high density polyethylene (HDPE) composites, Express Polym. Lett. 5 (2011) 581-590. doi:10.3144/expresspolymlett.2011.57.

[8] L.Z. Zhang, X.J. Wang, Y.Y. Quan, L.X. Pei, Conjugate heat conduction in filled composite materials considering interactions between the filler and base materials, Int. J. Heat Mass Transf. 64 (2013) 735-742. doi:10.1016/j.ijheatmasstransfer.2013.05.021.

[9] X.J. Wang, L.Z. Zhang, L.X. Pei, Thermal conductivity augmentation of composite polymer materials with artificially controlled filler shapes, J. Appl. Polym. Sci. 131 (2014) 1-10. doi:10.1002/app.39550.

[10] J.-Z. Liang, Thermal conductivity of $\mathrm{PP} / \mathrm{Al}(\mathrm{OH}) 3 / \mathrm{Mg}(\mathrm{OH}) 2$ composites, Compos. Part B Eng. 44 (2013) 248-252. doi:10.1016/j.compositesb.2012.05.033. 
[11] a. Neiman, E. Tsipis, I. Beketov, Y. Kotov, a. Murzakaiev, O. Samatov, Solid state interactions in nano-sized oxides, Solid State Ionics. 177 (2006) 403-410. doi:10.1016/j.ssi.2005.10.028.

[12] S.M. Ha, H.L. Lee, S.-G. Lee, B.G. Kim, Y.S. Kim, J.C. Won, et al., Thermal conductivity of graphite filled liquid crystal polymer composites and theoretical predictions, Compos. Sci. Technol. 88 (2013) 113-119. doi:10.1016/j.compscitech.2013.08.022.

[13] H. Zhou, S. Zhang, M. Yang, The effect of heat-transfer passages on the effective thermal conductivity of high filler loading composite materials, Compos. Sci. Technol. 67 (2007) 1035-1040. doi:10.1016/j.compscitech.2006.06.004.

[14] L.Z. Zhang, X.J. Wang, L.X. Pei, Nonlinear programming optimization of filler shapes for composite materials with inverse problem technique to maximize heat conductivity, Int. J. Heat Mass Transf. 55 (2012) 7287-7296. doi:10.1016/j.ijheatmasstransfer.2012.07.059.

[15] Z. Hashin, S. Shtrikman, A variational approach to the theory of the elastic behaviour of multiphase materials, J. Mech. Phys. Solids. 11 (1963) 127-140. doi:10.1016/00225096(63)90060-7.

[16] Z. Hashin, S. Shtrikman, A Variational approach to the theory of the effective magnetic permeability of multiphase materials, J. Appl. Phys. 33 (1962) 3125-3131. doi:10.1063/1.1728579.

[17] W. KW, Explanation of the dielectric fatigue phenomenon on the basis of Maxwell's concept, Arch. Fur Elektrotechnik. (1914).

[18] B. Weidenfeller, M. Höfer, F. Schilling, Thermal and electrical properties of magnetite filled polymers, Compos. Part A Appl. Sci. Manuf. 33 (2002) 1041-1053. doi:10.1016/S1359-835X(02)00085-4.

[19] F.R. Schilling, G.M. Partzsch, Quantifying partial melt fraction in the crust beneath the central andes and the tibetan plateau, 26 (2001) 239-246.

[20] W.J. Parker, R.J. Jenkins, C.P. Butler, G.L. Abbott, Flash method of determining thermal diffusivity, heat capacity, and thermal conductivity, J. Appl. Phys. 32 (1961) 1679-1684. doi:10.1063/1.1728417.

[21] W. Yi, L. Lu, D. Zhang, Linear Specific heat of carbon nanotubes, Phys. Rev. B. 59 (1999) R9015-R018.

[22] A. a Balandin, S. Ghosh, W. Bao, I. Calizo, D. Teweldebrhan, F. Miao, et al., Superior Thermal Conductivity of Single-Layer Graphene 2008, Nano Lett. 8 (2008) 902-907. doi:10.1021/n10731872. 
[23] M. Mohammad, K.I. Winey, Polymer Nanocomposites Containing Carbon Nanotubes, Macromolecules. 39 (2006) 5194-5205. doi:10.1021/ma060733p.

[24] F.P. Incropera, D.P. DeWitt, Fundamentals of Heat and Mass Transfer, 1981.

[25] a. Moisala, Q. Li, I. a. Kinloch, a. H. Windle, Thermal and electrical conductivity of single- and multi-walled carbon nanotube-epoxy composites, Compos. Sci. Technol. 66 (2006) 1285-1288. doi:10.1016/j.compscitech.2005.10.016. 